\title{
Cross sectional analysis of Chicago vs suburban Cook County suicide deaths among 10-24-year-olds in the Illinois violent death reporting system
}

Ernika G. Quimby ${ }^{1 *}$, Suzanne G. McLone ${ }^{2}$, Maryann Mason² and Karen Sheehan²

From 22nd Annual Injury Free Coalition for Kids ${ }^{\circledR}$ Conference: Forging New Frontiers: Moving Forward with Childhood Injury Prevention

Fort Lauderdale, Florida, USA. 01-03 December 2017

\begin{abstract}
Background: In 2014, suicide was the second leading cause of death among 10- to 24-year-olds in the US. Studies note disparities in youth suicide based on sex, race/ethnicity, and urban vs rural settings. This study investigates demographics, mental health indicators, and other circumstances surrounding youth/young adult deaths by suicide, comparing Chicago and suburban Cook County from 2005 to 2010.

Methods: Using the Illinois Violent Death Reporting System (IVDRS), we employed a cross-sectional design to provide descriptive analysis of decedents in three age groups (10-14, 15-19, and 20-24 years) in two geographic areas: urban (city of Chicago) and suburban (suburban Cook County) between January 1, 2005 and December 31, 2010. We used chi-square testing to test for significant differences in each age group by demographics, mental health indicators, and suicide markers in each area.
\end{abstract}

Results: Between 2005 and 2010, the IVDRS reported 299 deaths by suicide among 10-24-year-olds, 52\% in Chicago, and $48 \%$ in suburban Cook County. Of these deaths, 5.7\%, 33.4\% and 60.9\% were ages 10-14, 15-19, and $20-24$ years, respectively. Non-Hispanic (NH) whites comprised $50.7 \%$ of the totals, NH Blacks 26.5\%, Hispanics 16.8\%, and Asians 5. 7\%. In Chicago, males were $84 \%$ of suicides and $62.7 \%$ in suburban Cook County among 15-19-year-olds $(p<0.05)$. White race was significantly different in 10-14-year-olds: $0 \%$ in Chicago, $54 \%$ in suburban Cook County $(p<0.05)$. Racial and ethnic differences in suicides among 15-19-year-olds in Chicago vs suburban Cook County were: NH White 22.4\% vs $74.5 \%(p<0.001)$, NH Black $46.9 \%$ vs $13.7 \%(p<0.05)$, Hispanic $24.5 \%$ vs $7.8 \%(p<0.05)$. There were also differences for $20-24$-year-olds with $\mathrm{NH}$ White $43 \%$ vs $65.4 \%$ and $\mathrm{NH}$ Black $32 \%$ vs $13.6 \%$ ( $p<0.05$ for both). For mechanism of death, in 15-19-year-olds, there were differences between city and suburban in firearm deaths ( $42.9 \%$ vs $20 \%, p<0.05$ ) and in poisoning ( 0 vs 14\%, $p<0.05$ ).

Conclusions: Our analyses detected significant location-related differences in the characteristics of decedents within the Chicago region indicating that local data are needed to inform suicide prevention efforts so that those at most risk can be prioritized for services. IVDRS is a potent tool in identifying these variations.

Keywords: Suicide, Young adults, Violent death

\footnotetext{
* Correspondence: quimbyeg@email.chop.edu

'Division of Emergency Medicine, The Children's Hospital of Philadelphia,

Philadelphia, PA, USA

Full list of author information is available at the end of the article
} 


\section{Background}

In 2014, suicide was the second leading cause of death among 10 to 24-year-olds in the United States (Centers for Disease Control and Prevention, 2018). In 2004, suicide was the third leading cause of death in this age group (Lubell et al., 2007). More specifically, WISQARS data show suicide rates significantly increased in the United States between 2005 and 2010 among 10-14year-olds, 15-19-year-olds and 20-24-year-olds, by 52\%, $28 \%$, and $22 \%$, respectively. Suicide has become an increasingly high cause of death in Illinois metropolitan areas (which include Cook County) among 10-14-yearolds, rising from the 8th leading cause of death in 2005, to fifth in 2010, and finally to the number one leading cause of death in 2015 for this age group. Among all youth aged 10-24 years in the Illinois metropolitan area, the suicide rate per 100,000 increased from $4.2 \%$ in 2005 to $5.6 \%$ in 2010 to $7.9 \%$ in 2015 . Significant increases have occurred during this time period for Non-Hispanic White youths in all three of these age groups, and in Non-Hispanic Black youths in 15-19-year-olds. These data indicate that suicide is a serious and persistent health risk for youth and young adults in the United States, and specifically in metropolitan areas in Illinois.

Studies have noted disparities in youth suicide based on sex, race/ethnicity and circumstances surrounding death, including location. These disparities point to the need for strategies targeting factors contributing to suicide deaths in youth/young adults, especially among disproportionately affected groups (Karch et al., 2013; McLone et al., 2016; Bridge et al., 2015; Fontanella et al., 2015). For example, national data sets have found most decedents are male (Karch et al., 2013). Age related differences have been documented, including differences in method of suicide death and association of a preceding crisis (McLone et al., 2016). With regard to racial disparities, between 1993 and 2012, though the overall suicide death rate among children aged 5-11 years was stable, suicide significantly increased for Black children and significantly decreased for White children (Bridge et al., 2015). There is also evidence that the setting in which youth live may be associated with suicidality trends. For example, urban-rural disparities, have also been identified, with studies finding higher youth and young adult suicide rates in rural communities (Fontanella et al., 2015). This study investigates demographics, mental health indicators, and other circumstances surrounding youth/young adult deaths by suicide, comparing Chicago and suburban Cook County from 2005 to 2010 to begin to better identify trends in recent years.

\section{Methods}

This study was cross-sectional, using the Illinois Violent Death Reporting System (IVDRS), a surveillance system containing information on violent deaths including suicide, homicide, legal intervention, and unintentional firearm deaths in Illinois. IVDRS collects data from death certificates, coroner/medical examiner, law enforcement, and toxicology reports to identify the circumstances surrounding each death (IVDRS 2015). The IVDRS is based on the National Violent Death Reporting System (NVDRS). The NVDRS is a state-based surveillance system developed by the Centers for Disease Control and Prevention, National Center for Injury Prevention and Control (CDC Injury Center). NVDRS collects data on violent deaths, including decedent demographics, mechanism of injury, method of injury, location of residence, location of injury, location of death, autopsy and toxicology results, and circumstances surrounding the death. The primary data sources are death certificates, coroner/ medical examiner reports, law enforcement reports and crime lab reports; data are linked by decedent and entered into a web-based data repository.

The IVDRS collects violent data from a sample of urban, suburban and rural counties in Illinois. From 2005 to 2014, the IVDRS operated as a shadow system as it used CDC Injury Center software and protocols to collect data, but no funding or official support was received from the CDC Injury Center. IVDRS data collected prior to January 1, 2015 are not in the CDC's Web-based Injury Statistics Query and Reporting System (WISQARS), which is an interactive, online database that provides fatal and nonfatal injury, violent death, and cost of injury data from a variety of trusted sources. From 2005 to 2010 the IVDRS only collected data from five Illinois counties, including Cook County, Illinois, which contains the City of Chicago. For the years 20112014 only some basic data elements were collected; therefore, data from these years were insufficient to be included in this present study.

The data used for this analysis included all data contained in IVDRS for calendar years 2005-2010, which represents all data collected on the decedents during this time period as of June 2014. Decedent cases were selected according to the following criteria: manner of death was suicide, decedents were between the ages of 10 and 24 years, and both the injury and subsequent death occurred in Cook County.

All decedent cases were separated into the following age groups, in years: $10-14,15-19$, and 20-24. We described demographics, method of suicide, and the presence of 13 separate circumstances among these age groups: Suicide occurred in own residence, positive alcohol test, problems with current or former intimate partner, other relationship problem(s) with family member/ friend/associate, problems at school, crisis within two weeks of suicide, current depressed mood (not a diagnosis), current mental health diagnosis, currently in 
treatment for a mental health issue, ever treated for a mental health issue (diagnosed), history of prior suicide attempt, disclosed intent to commit suicide, and presence of a suicide note. All variables were described using percentages. Differences between urban (City of Chicago) and suburban (remainder of Cook County) were assessed using Pearson's $\chi^{2}$.

Data were collected in software developed by the CDC for NVDRS; all analyses were performed using SPSS 22.0 (IBM, Chicago, IL).

The study was deemed exempt from Institutional Review Board review.

\section{Results}

Between 2005 and 2010, IVDRS reported 299 deaths by suicide among 10-24-year-olds, 52\% of whom were in Chicago, and $48 \%$ in suburban Cook County. NonHispanic whites comprised $51 \%$ of the totals, NonHispanic Blacks 26\%, Hispanics 17\%, and Asians 6\%. Hanging/strangulation was the most common mechanism of death at $44 \%$, followed by firearm (29\%) and poisoning (12\%). Amongst the 299 suicide deaths, 6\%, 33\% and $61 \%$ were ages $10-14,15-19$, and $20-24$ years, respectively. We compared Chicago and suburban Cook County within each of the three age groups.

Demographic characteristics included sex and race/ ethnicity (Table 1). The majority of suicide deaths in both the Chicago and suburban groups were males across all age groups. However, male/female percentages were significantly different between Chicago and suburban Cook County. In Chicago, males made up $84 \%$ of suicides, and $63 \%$ in suburban Cook County, among 15-19-year-olds $(p<0.05)$.
There was significant variation in the proportion of decedents' race/ethnicity between the urban (Chicago) and suburban (suburban Cook County) locations, in all three age groups. The proportion of decedents who were nonHispanic White was significantly different between the two locations among 10-14-year-olds, with 0\% in Chicago and $54 \%$ in suburban Cook County $(p<0.05)$. Among 15 19-year-olds, the proportion of decedents by race/ethnicity for non-Hispanic (NH) Whites, $\mathrm{NH}$ Blacks, and Hispanics were each significantly different between Chicago and suburban Cook County (NH White $22 \%$ vs $74 \%, p<0.05$; NH Black $47 \%$ vs $14 \%, p<0.05$; Hispanic $24 \%$ vs $8 \%, p<0.05)$. Finally, statistically significant differences were found for the proportion of NH White (43\% vs $65 \%$ for Chicago and suburban Cook County, respectively) and NH Black (32\% vs 14\%) ( $p<0.05$ for both) decedents among 20-24-year-olds.

Hanging/strangulation, firearms, and poisoning, in that order, were the most common means/methods of suicide deaths in all three of the age groups, in both Chicago and suburban Cook County (Table 1). While no statistically significant differences between locations were found for method of death in ages 10-14 years, there were significant differences in the means of death between locations in the other two age groups. In 15-19-year-olds, there were statistically significant differences between Chicago and suburban Cook County in firearm deaths $(43 \%$ vs $20 \%, p<0.05)$ and in poisoning deaths ( $0 \%$ vs $14 \%$, $p<0.05$ ). Finally, in 20-24-year-olds, only firearm deaths were significantly different between Chicago and suburban Cook county (33\% vs $25 \%, p<0.05)$.

Results of the circumstances surrounding each death are presented in Table 2 . There were no statistically significant differences between the two locations across any

Table 1 Participant characteristics by location and age group

\begin{tabular}{|c|c|c|c|c|c|c|c|c|c|}
\hline \multirow[b]{4}{*}{ Variable } & \multicolumn{2}{|l|}{10 to 14} & & \multicolumn{2}{|l|}{15 to 19} & & \multicolumn{2}{|l|}{20 to 24} & \\
\hline & \multicolumn{2}{|l|}{$n=17$} & & \multicolumn{2}{|l|}{$n=100$} & & \multicolumn{2}{|l|}{$n=182$} & \\
\hline & Chicago & Suburban Cook & & Chicago & Suburban Cook & & Chicago & Suburban Cook & \\
\hline & $\%$ & $\%$ & & $\%$ & $\%$ & & & & \\
\hline \multicolumn{10}{|l|}{ Sex } \\
\hline Male & 66.7 & 81.8 & & 83.7 & 62.7 & $p<0.05$ & 80.2 & 81.5 & \\
\hline Female & 33.3 & 18.2 & & 16.3 & 37.3 & & 19.8 & 18.5 & \\
\hline \multicolumn{10}{|l|}{ Race/Ethnicity } \\
\hline NH White & 0.0 & 54.5 & $p<0.05$ & 22.4 & 74.5 & $p<0.05$ & 43.0 & 65.4 & $p<0.05$ \\
\hline NH Black & 50.0 & 27.3 & & 46.9 & 13.7 & $p<0.05$ & 32.0 & 13.6 & $p<0.05$ \\
\hline Hispanic & 50.0 & 18.2 & & 24.5 & 7.8 & $p<0.05$ & 18.0 & 13.6 & \\
\hline \multicolumn{10}{|l|}{ Means/Method } \\
\hline Firearm & 16.7 & 9.1 & & 42.9 & 20.0 & $p<0.05$ & 33.0 & 25.0 & $p<0.05$ \\
\hline Hanging/Strangulation & 83.3 & 72.7 & & 40.8 & 52.0 & & 33.0 & 48.8 & \\
\hline Poison & 0.0 & 9.1 & & 0.0 & 14.0 & $p<0.05$ & 13.0 & 18.8 & \\
\hline
\end{tabular}


Table 2 Circumstances surrounding suicide and factors contributing to suicide by location and age group

\begin{tabular}{|c|c|c|c|c|c|c|}
\hline \multirow[b]{4}{*}{ Variable } & \multicolumn{2}{|l|}{10 to 14} & \multicolumn{2}{|l|}{15 to 19} & \multicolumn{2}{|l|}{20 to 24} \\
\hline & \multicolumn{2}{|l|}{$n=17$} & \multicolumn{2}{|l|}{$n=100$} & \multicolumn{2}{|l|}{$n=182$} \\
\hline & Chicago & Suburban Cook & Chicago & Suburban Cook & Chicago & Suburban Cook \\
\hline & $\%$ & $\%$ & $\%$ & $\%$ & $\%$ & $\%$ \\
\hline \multicolumn{7}{|l|}{ Circumstances } \\
\hline Occurred at decedent's residence & 83.3 & 81.8 & 65.3 & 70.6 & 58.4 & 66.7 \\
\hline Positive for alcohol & 0.0 & 0.0 & 16.7 & 30.6 & 42.1 & 46.4 \\
\hline \multicolumn{7}{|l|}{ Factors which appeared to have contributed to suicide } \\
\hline Problems with current/former intimate partner & 0.0 & 9.1 & 22.4 & 15.7 & 26.7 & 16.0 \\
\hline Other relationship problem(s) & 33.3 & 36.4 & 18.4 & 17.6 & 6.9 & 7.4 \\
\hline Problems at school & 16.7 & 27.3 & 4.1 & 2.0 & 1.0 & 4.9 \\
\hline Victim experienced crisis within 2 weeks of suicide & 33.3 & 27.3 & 14.3 & 13.7 & 21.8 & 21.0 \\
\hline
\end{tabular}

age groups with regard to whether the incident occurred at the decedent's residence (the majority did in all groups, though the percentage decreased in both locations among the older ages) or whether the decedent tested positive for alcohol (the majority did not in all groups, though the percentage increased in both locations among the older ages). Several other factors were investigated, including whether the decedent had problems with a current or former intimate partner, other relationship problem(s) with a family member/friend/ associate, problems at school, or if he or she had experienced a crisis within two weeks of suicide, but none were significantly different between Chicago and suburban Cook County in any of the age groups.

Multiple mental health indicators, including diagnosed mental health problems and noted, but not necessarily diagnosed, depressed mood, were analyzed (Table 3). In 20-24-year-olds, in Chicago, 29\% had a current diagnosed mental health problem compared with $47 \%$ in suburban Cook County $(p<0.05)$. In $10-14$-year-olds, two variables were significant: currently or history of ever being treated for a mental health problem (both variables were $0 \%$ in Chicago vs $36 \%$ in suburban Cook County, $p<0.05$ ). Current depressed mood did not show any significant difference. We also analyzed specific suicide concerns. The decedent disclosing an intent to commit suicide to another person was significantly different between Chicago and suburban Cook County only among 10-14-year-olds ( $0 \%$ vs $36 \%, p<0.05)$. The decedent leaving a suicide note was significantly different between Chicago and suburban Cook County only among $15-19$-year-olds (22\% vs $45 \%$, $p<0.05)$. There was no significant difference between the two locations found for decedents having a history of prior suicide attempts. The majority of decedents, across both locations and ages, did not have a recorded mental health or suicide history.

Table 3 Mental health and suicide indicators by location and age group

\begin{tabular}{|c|c|c|c|c|c|c|c|c|c|}
\hline \multirow[b]{4}{*}{ Variable } & \multicolumn{2}{|l|}{10 to 14} & & \multicolumn{2}{|l|}{15 to 19} & & \multicolumn{2}{|l|}{20 to 24} & \\
\hline & \multicolumn{2}{|l|}{$n=17$} & & \multicolumn{2}{|l|}{$n=100$} & & \multicolumn{2}{|l|}{$n=182$} & \\
\hline & Chicago & Suburban Cook & & Chicago & Suburban Cook & & Chicago & Suburban Cook & \\
\hline & $\%$ & $\%$ & & $\%$ & $\%$ & & $\%$ & $\%$ & \\
\hline Mental Health & 0.0 & 27.3 & & 38.8 & 21.6 & & 20.8 & 24.7 & \\
\hline \multicolumn{10}{|l|}{$\begin{array}{l}\text { Current depressed mood } \\
\text { (not a diagnosis) }\end{array}$} \\
\hline Current mental health diagnosis & 16.7 & 45.5 & & 36.7 & 35.3 & & 28.7 & 46.9 & $p<0.05$ \\
\hline $\begin{array}{l}\text { Currently in treatment for mental } \\
\text { health diagnosis }\end{array}$ & 0.0 & 36.4 & $p<0.05$ & 24.5 & 29.4 & & 13.9 & 21.0 & \\
\hline $\begin{array}{l}\text { History of ever being treated for } \\
\text { mental health issue (diagnosed) }\end{array}$ & 0.0 & 36.4 & $p<0.05$ & 28.6 & 33.3 & & 18.8 & 29.6 & \\
\hline \multicolumn{10}{|l|}{ Suicide } \\
\hline History of prior suicide attempts & 16.7 & 18.2 & & 16.3 & 13.7 & & 18.8 & 14.8 & \\
\hline Disclosed intent to commit suicide & 0.0 & 36.4 & $p<0.05$ & 20.4 & 25.5 & & 11.9 & 16.0 & \\
\hline Suicide note & 0.0 & 18.2 & & 22.4 & 45.1 & $p<0.05$ & 22.8 & 24.7 & \\
\hline
\end{tabular}




\section{Discussion}

Overall, we found several significant differences by race/ ethnicity, sex, and mental health indicators between the urban and suburban location for each youth age group. These findings have implications for how to best tailor suicide prevention services to distinct populations taking into account race/ethnicity, age and location by highlighting different features that could potentially be used to identify and target high risk youth populations for services, as the need for further research and identification of trends continues (Gould et al., 2003). Additionally, prior studies have indicated that though both parents and youth recognize that suicide is an important problem, they often think that it is a problem in other communities, not their own (Schwartz et al., 2010). Identification of common features more specific to communities could thus help personalize information presented to parents and youth themselves, not just those providing services.

In our findings, across age groups, there were significantly more White decedents in the suburban setting. Among older decedents aged 15-19-years old, there were significantly more Blacks and Hispanics in the city setting, and additionally more Black decedents in the city among 20-24-year-olds. Multiple theories have been described for this, including increased stress on youth of color relating to racism, and a lack of access to resources at the individual and family levels; a "double risk of death" due to high rates of homicide and suicide amongst Black men also emphasizes the need for more identification and intervention of risk (Baker, 1990).

Method of death warrants further discussion from a preventive stance. In our study, firearm deaths were more common in the city than suburban settings, with statistical difference found in older youth above 15 years old. More research is needed to better elucidate related factors, such as access to guns in the different settings, as well as storage of guns in homes that do have them. Studies have shown a significantly increased risk of suicide when guns are accessible, even when controlling for confounders (Miller et al., 2007; Birckmayer \& Hemenway, 2001). The increased risk of suicide death with gun access may be even higher among youth than older adults; for example, one study showed a statistically significant increase for 15-24-year olds after controlling for such factors as education, poverty, and unemployment, but the same relationship was not seen among 25-44 year olds (Birckmayer \& Hemenway, 2001). In terms of access, another study highlighted that the majority of adolescent suicide deaths are committed using a family member's gun (Johnson et al., 2010). Prior research has indicated that among households that have a gun, the risk of both intentional and nonintentional injuries may be reduced with safe-storage methods, with the gun locked and unloaded with ammunition stored locked in a different location (Grossman et al., 2005).

Although no differences were noted between the groups' circumstances and surrounding factors, prior studies indicate an association between youth suicide and factors such as comorbid alcohol abuse or family discord (Cash \& Bridge, 2009). Several more specific mental health indicators did reveal differences. Suburban youth in the youngest age group, 10-14year-olds, were more likely to be currently or previously receiving treatment for a mental health issue than urban children in the same age group. This may be related to the lack of mental health services for youth, especially in locations with limited resources, and highlights the need for providers besides specialized mental health practitioners, such as pediatricians to assess suicide risk in youth with whom they interact (Shain, 2007).

Limitations of this study include the small sample size that was present in the database. Additionally, subjects were grouped into two large categories "Chicago" and "Suburban Cook County", though both areas have significant diversity within each subgroup. The age of the data is another limitation; due to the constraints of the data available through the IVDRS, only data through 2010 was available for use. The mental health indicator, "Current depressed mood" is not a diagnosis and is dependent on next of kin reporting. This may lead to under reporting of depressed mood. The data were all retrospectively obtained through the IVDRS database, for deaths determined to be suicides. Data abstractors are limited to the data included in the reports they receive. Reports might not fully reflect all information known about an incident. Although extensive coding training is conducted and help desk support is available daily, variations in coding might occur depending on the abstractor's level of experience. For this reason, IVDRS regularly conducts blinded reabstraction of cases to test consistency and identify training needs. Protective factor data (i.e., characteristics or circumstances that reduce the risk for violent death) are not collected by NVDRS as a result of the nature of death certificate, CME record, and police reports, which typically contain only circumstances associated with risk factors. Finally, medical and mental health information (e.g., type of condition and whether the decedent was currently receiving treatment) are not often captured directly from medical records but from coroner/medical examiner reports and the decedent's family members and friends. Therefore, the completeness and accuracy of this information are limited by the knowledge of the informant (Karch et al., 2009; Lyons et al., 2016). 


\section{Conclusion}

Our analyses detected significant differences by location among youth suicide decedents within the Chicago region. These findings indicate that local data, such as the IVDRS, may help to inform suicide prevention efforts by identifying high risk populations of youth within specific regions. Future research is warranted to further explore reasons for these differences in demographic and situational circumstances for youth suicide to better inform local prevention efforts. Additionally, specific to the IVDRS, research should identify additional qualitative findings to further elucidate patterns of suicide in urban and suburban Chicago settings.

\section{Abbreviations}

IVDRS: Illinois Violent Death Reporting System; NVDRS: National Violent Death Reporting System

\section{Consent for publications}

Not applicable.

\section{Funding}

Publication of this article was funded by Grant Number 6 NU17CE002590-04-01, funded by the Centers for Disease Control and Prevention. Its contents are solely the responsibility of the authors and do not necessarily represent the official views of the Centers for Disease Control and Prevention or the Department of Health and Human Services. Additional support also provided by the Illinois Department of Public Health and an anonymous donor. Publication of this article was funded by the Injury Free Coalition for Kids ${ }^{\oplus}$.

\section{Availability of data and materials}

Data will not be shared due to data ownership agreements.

\section{About this supplement}

This article has been published as part of Injury Epidemiology Volume 5 Supplement 1, 2018: Proceedings from the 22nd Annual Injury Free Coalition for Kids ${ }^{\circledast}$ Conference: Forging New Frontiers: Moving Forward with Childhood Injury Prevention. The full contents of the supplement are available online at https://injepijournal.springeropen.com/articles/ supplements/volume-5-supplement-1.

\section{Authors' contributions}

EQ and SM conducted the literature search and data interpretation and wrote the manuscript. SM performed the data analysis. MM and KS provided further review of the data and interpretation. All authors read and approved the final manuscript.

\section{Ethics approval and consent to participate}

Not applicable.

\section{Consent for publication}

Not applicable.

\section{Competing interests}

The authors declare that they have no competing interests.

\section{Publisher's Note}

Springer Nature remains neutral with regard to jurisdictional claims in published maps and institutional affiliations.

\section{Author details}

'Division of Emergency Medicine, The Children's Hospital of Philadelphia, Philadelphia, PA, USA. ${ }^{2}$ Injury Prevention and Research Center, Ann \& Robert H. Lurie Children's Hospital of Chicago, Chicago, IL, USA.
Published: 10 April 2018

\section{References}

Baker FM. Black youth suicide: literature review with a focus on prevention. J Natl Med Assoc. 1990;82(7):495-507.

Birckmayer J, Hemenway D. Suicide and gun prevalence: are youth disproportionately affected? Suicide Life Threat Behav. 2001;31:303-10.

Bridge JA, Asti L, Horowitz LM, Greenhouse JB, Fontanella CA, Sheftall AH, Kelleher KJ, Campo JV. Suicide trends among elementary school-aged children in the United States from 1993 to 2012. JAMA Pediatr. 2015;169(7):673-7.

Cash SJ, Bridge JA. Epidemiology of youth suicide and suicidal behavior. Curr Opin Pediatr. 2009;21(5):613-9.

Centers for Disease Control and Prevention. WISQARS Leading Causes of Death Reports, National and Regional, 1999-2014. National Center for Health Statistics, National Vital Statistics System. http://webappa.cdc.gov/sasweb/ ncipc /leadcaus10_us.html. Accessed 19 Sept 2016.

Fontanella CA, Hiance-Steelesmith DL, Phillips GS, Bridge JA, Lester N, Sweeney HA, Campo JV. Widening rural-urban disparities in youth suicides, United States, 1996-2010. JAMA Pediatr. 2015;169(5):466-73.

Gould MS, Greenberg T, Velting DM, Shaffer D. Youth suicide risk and preventive interventions: a review of the past 10 years. J Am Acad Child Adolesc Psychiatry. 2003:42:386-405.

Grossman DC, Mueller BA, Riedy C, Dowd MD, Villaveces A, Prodzinski J, Nakagawara J, Howard J, Thiersch N, Harruff R. Gun storage practices and risk of youth suicide and unintentional firearm injuries. JAMA. 2005;293(6):707-14.

Johnson RM, Barber C, Azrael D, Clark DE, Hemenway D. Who are the owners of firearms used in adolescent suicides? Suicide Life Threat Behav. 2010;40:609-11.

Karch DL, Dahlberg LL, Patel N, Davis TW, Logan JE, Hill HA, Ortega L. Surveillance for violent deaths - National Violent Death Reporting System, 16 states, 2006. MMWR Surveill Summ. 2009;58(11):1-44.

Karch DL, Logan J, McDaniel DD, Floyd CF, Vagi KJ. Precipitating circumstances of suicide among youth aged 10-17 years by sex: data from the National Violent Death Reporting System, 16 states, 2005-2008. J Adol Health. 2013;53:551-3.

Lubell KM, Kegler SR, Crosby AE, Karch D. Centers for Disease Control and Prevention. Suicide trends among youths and young adults aged 10-24 years, United States:1990-2004. MMWR Morb Mortal Wkly Rep. 2007;56(35):905-8.

Lyons BH, Fowler KA, Jack SP, Betz CJ, Blair JM. Surveillance for violent deaths National Violent Death Reporting System, 17 states, 2013. MMWR Surveill Summ. 2016;65(10):1-42.

McLone SG, Loharikar A, Sheehan K, Mason M. Suicide in Illinois, 2005-2010: a reflection of patterns and risks by age groups and opportunities for targeted prevention. J Trauma Acute Care Surg. 2016;81(4):S30-5.

Miller M, Lippmann SJ, Azrael D, Hemenway D. Household firearm ownership and rates of suicide across U.S. states. J Trauma. 2007:62:1029-35.

Schwartz KA, Pyle SA, Dowd MD, Sheehan K. Attitudes and beliefs of adolescents and parents regarding adolescent suicide. Pediatrics. 2010;125(2):221-7.

Shain BN. American Academy of Pediatrics, committee on adolescence. Suicide and suicide attempts in adolescents. Pediatrics. 2007:120(3):669-76.

\section{Submit your manuscript to a SpringerOpen ${ }^{\circ}$ journal and benefit from:}

- Convenient online submission

- Rigorous peer review

- Open access: articles freely available online

- High visibility within the field

- Retaining the copyright to your article

Submit your next manuscript at $>$ springeropen.com 